\section{Pruebas diagnósticas no validadas para alergia alimentaria}

\section{Non validated diagnostic tests for food allergy}

\section{Señor Editor,}

El diagnóstico de alergia alimentaria se basa fundamentalmente en una historia clínica detallada y examen físico minucioso. De acuerdo al tipo de cuadro clínico del paciente, algunas pruebas clínicas y exámenes de laboratorio son utilizados como herramientas de apoyo diagnóstico. El tratamiento depende de las características clínicas y otras variables que deben ser consideradas cuidadosamente por el médico tratante.

En base a la evidencia disponible a la fecha, las pruebas validadas para el estudio de alergias alimentarias son: prick test, IgE específicas, test de parches sólo en algunos cuadros específicos, y el test de provocación oral ${ }^{1}$.

Múltiples pruebas no convencionales (como por ejemplo NAET, ALCAT y biorresonancia, entre otras) han sido planteadas como nuevos métodos diagnósticos, sin embargo, carecen de plausibilidad biológica, no están estandarizadas, y su utilidad no ha sido demostrada en estudios clínicos adecuados para este fin $^{2}$. Es importante informar a los pacientes los potenciales riesgos derivados del uso de estas técnicas no validadas por la literatura, con especial énfasis en las extensas restricciones alimentarias que se suelen indicar en base a sus resultados. Los consensos internacionales en alergia alimentaria no recomiendan estas pruebas como herramientas complementarias ni alternativas, e incluso desaconsejan su uso ${ }^{1}$.

Tomando en consideración estos precedentes, la Sociedad Chilena de Alergia e Inmunología no valida el uso de pruebas diagnósticas no convencionales en el diagnóstico de alergia alimentaria.

Daniela Cereceda ${ }^{1}$, Rocío Tordecilla ${ }^{1}$, Raquel Aguilera $^{1}$, Ana María Gallardo', Masumi Grau ${ }^{1}$, Pablo Herrera ${ }^{1}$, Lorena Jiusán ${ }^{1}$, Macarena Lagos ${ }^{1}$, Patricia Roessler', Pamela Hernández ${ }^{1}$, Pablo Raby ${ }^{1}$ ${ }^{1}$ Sociedad Chilena de Alergia e Inmunología.

\section{Referencias}

1. Muraro A, Werfel T, Hoffmann-Sommergruber K, Roberts G, Beyer K, Bindslev-Jensen C, et al. EAACI food allergy and anaphylaxis guidelines: diagnosis and management of food allergy. Allergy 2014; 69 (8): 1008-25.

2. Kelso JM. Unproven Diagnostic Tests for Adverse Reactions to Foods. J Allergy Clin Immunol Pract 2018; 6 (2): 362-5.

Correspondencia a:

Dr. Pablo Raby

Pedro Canisio 1103 dpto 32, Vitacura.

Santiago, Chile.

pablo.raby@gmail.com 